\title{
Volumetric MRI measurements of the tree shrew hippocampus
}

\author{
F. Ohl ${ }^{\text {a }}$, T. Michaelis ${ }^{\text {b }}$, H. Fujimori ${ }^{\text {b }}$, J. Frahm ${ }^{\text {b }}$, S. Rensing ${ }^{\text {a }}$, E. Fuchs ${ }^{\text {a,* }}$ \\ a Division of Neurobiology, German Primate Center, Kellnerweg 4, 37077 Göttingen, Germany \\ ${ }^{\mathrm{b}}$ Biomedizinische NMR Forschungs GmbH am Max-Planck-Institut für Biophysikalische Chemie, 37070 Göttingen, Germany
}

Received 20 August 1998; received in revised form 3 February 1999; accepted 5 February 1999

\begin{abstract}
Protocols suitable for repeated magnetic resonance imaging (MRI) studies of the tree shrew's brain were established. This included the development of (i) a technique for prolonged inhalation anesthesia by endotracheal intubation; (ii) a reproducible fixation of the animal's head in a stereotaxic frame and finally (iii) the set-up of the hardware (rf coil) and software (MRI sequences) of the MRI system. The endotracheal intubation as well as the repeated and prolonged anesthesia showed no complications. The in vivo measurements of the tree shrew's hippocampal formation revealed a high reproducibility. Right and left hippocampal volume was determined as $85.2 \mathrm{~mm}^{3} \pm 8 \%$ and $87.4 \mathrm{~mm}^{3} \pm 10 \%$, respectively. The utility of MRI in delineating alterations in brain anatomy was demonstrated in three animals receiving cortisol via the drinking water (5 mg/animal/day). After a 4-week treatment, in two of the three tree shrews a reduction in hippocampal volume was observed. Thus, the MRI protocols used here allow for repeated and non-invasive measurements of changes in hippocampal anatomy within the same animal and to monitor the temporal dynamics of structural alterations within this brain structure. (C) 1999 Elsevier Science B.V. All rights reserved.
\end{abstract}

Keywords: Brain MRI; Cortisol; Hippocampal atrophy; Hippocampal volume; Tree shrew

\section{Introduction}

Most available techniques in neurobiology are limited by their invasiveness which precludes repeated measurements of the same animal. Magnetic resonance imaging (MRI) can overcome these disadvantages and offers new perspectives for neuroscientific research (Ting and Bendel, 1992; Frahm, 1993; Vullo et al., 1996). In humans, neuroimaging is one important tool to evaluate degenerative processes which lead to volumetric changes of certain brain structures. There is increasing evidence that various neuropathologies, such as schizophrenia (Fukuzako et al., 1996), depression (O'Brian et al., 1996), Alzheimer's disease (Jack et al., 1992), as well as aging (Lupien et al., 1998) are associ-

\footnotetext{
* Corresponding author. Tel.: + 49-551-3851 130; fax.: + 49-5513851228.

E-mail address: efuchs@gwdg.de (E. Fuchs)
}

ated with hippocampal atrophy, resulting in the volumetric reduction of this brain structure. The hippocampal formation is of crucial importance for cognitive processes (Eichenbaum et al., 1992; Squire, 1992; Tulving and Markowitsch, 1997) and alterations of this brain area are associated with an impairment of memory performance (McEwen 1997; Schacter, 1997; Lupien et al. 1998). Since the underlying mechanisms of the neuronal alterations are not well understood there is a great interest to establish repetitive neuroimaging techniques investigating the temporal dynamics of structural alterations in animal models which mimic these neuropathological processes.

Tree shrews have been shown to be a valuable animal model to investigate the neuroendocrine and behavioral consequences of chronic psychosocial stress (Fuchs et al., 1996). Recent studies in chronically stressed tree shrews have demonstrated both a high face and a predictive validity for depression (Fuchs et al., 1996). 
For chronically stressed tree shrews we further could demonstrate structural alterations of the hippocampal pyramidal neurons (Magariños et al., 1996), suppression of neurogenesis in the dentate gyrus (Gould et al., 1997), and impaired memory abilities (Ohl and Fuchs, 1998).

The purpose of this study was to establish protocols suitable for repeated MRI studies of the tree shrew's brain, thus allowing measurements of the hippocampal volume in the same animal during different stages of an experiment. Whether the histological changes observed during chronic stress are translated into measurable changes in hippocampal volume we treated animals during 4 weeks with the adrenocortical steroid hormone cortisol via the drinking water and measured the hippocampal volume at the end, and 7 and 12 weeks after the treatment. Since controllable and prolonged anesthesia is a prerequisite for MRI studies in animals, we developed a technique for prolonged inhalation anesthesia. The method of choice to deliver inhalation anesthetics to animals is via an endotracheal tube. This ensures the delivery of the agent at the required concentration directly into the respiratory tract and allows direct inhalation. The difficulty of the technique and lack of commercially available equipment for smaller animals such as tree shrews (body weight of about 200 g) has discouraged the use of endotracheal intubation (Flecknell, 1996). However, successful prolonged inhalation anesthesia has been reported for small primates (Morris et al., 1997), rodents (Flecknell, 1996), and tree shrews (Ter Laak et al., 1975) using purpose made equipments. To ensure a reproducible fixation of the animal's head a stereotaxic frame was developed. The frame was entirely made of plastic, and allowed a highly differentiated fixation of every individual's head. Finally, we addressed the set-up of the hardware ( $\mathrm{rf}$ coil) and software (MRI sequences) of the MRI system.

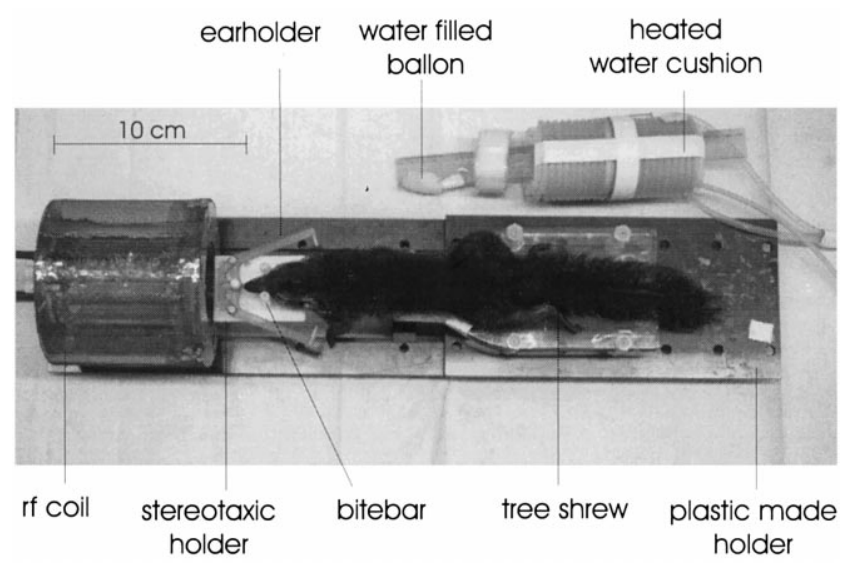

Fig. 1. Tree shrew placed in a prone position on the stereotaxic holder.

\section{Methods}

\subsection{Animals and housing}

Adult male tree shrews (Tupaia belangeri; $n=10$; body weight $170-250 \mathrm{~g}$ ) were from the breeding colony at the German Primate Center (Göttingen, Germany). Animals were individually housed on a regular day/ night cycle with artificial illumination from 08:00 to 20:00 $\mathrm{h}$ in air-conditioned rooms with water and food ad lib (Tupaia Diet, Altromin, Lage, Germany; for details of housing conditions see Fuchs and Schumacher, 1990). The animal experiments were approved by the Government of Lower Saxony, Germany.

\subsection{Anesthesia}

Anesthesia was induced by i.m. injection of xylazin ( 2 $\mathrm{mg} / \mathrm{kg})$, ketamine $(10 \mathrm{mg} / \mathrm{kg})$, atropine $(0.02 \mathrm{mg} / \mathrm{kg})$ immediately after removing the animal from its cage to minimize possible stressful effects by transportation to the MRI room. After being completely relaxed, the animals were intubated under visual control (laryngoscope, Aesculap AN404) with a purpose made endotracheal tube (ED $2.0 \mathrm{~mm}$ ). When passed into the trachea, the tube was secured with an adhesive tape and connected to the anesthetic circuit $\left(70: 30 \quad \mathrm{~N}_{2} \mathrm{O}: \mathrm{O}_{2}\right.$ with $0.8-1.5 \%$ halothane). Body temperature was maintained constant using a heated water cushion $\left(30^{\circ} \mathrm{C}\right)$. To control both breathing frequency and amplitude a water filled balloon was attached to the animals' chest and respiration was monitored (Fig. 1).

\subsection{Stereotaxic frame}

After inducing anesthesia and intubating the trachea the animal was placed in a prone position on a plastic holder (Fig. 1). The stereotaxic frame allowed reproducible fixation of the head. The mouth of the animals was put on a bite-bar which was fixed on the holder. The bite-bar was placed and fixed right behind the incisors. Finally, the position of the head was optimized by earholders. This resulted in a centered position of the brain in the rf coil as well as in the magnet.

\subsubsection{Magnetic resonance imaging (MRI)}

MRI was carried out at a field strength of $2.35 \mathrm{~T}$ using a MRBR 4.7/400 mm magnet (Magnex Scientific, Abingdon, UK) driven by an ABX-11 system (Bruker, Karlsruhe, Germany). A homebuilt birdcage coil (ID 60 $\mathrm{mm}$ ) was used for homogenous rf excitation and reception of the MRI signal. Sagittal and coronal multi-slice gradient-echo images (rf-spoiled FLASH, field of view $50 \mathrm{~mm}$, matrix $256 \times 256$, nine slices, section thickness $1 \mathrm{~mm}$ with $0.5 \mathrm{~mm}$ gap, two averages, $150 \mathrm{~ms}$ repetition time, $5 \mathrm{~ms}$ echo time, and $20^{\circ}$ flip angle) were obtained 
(a)

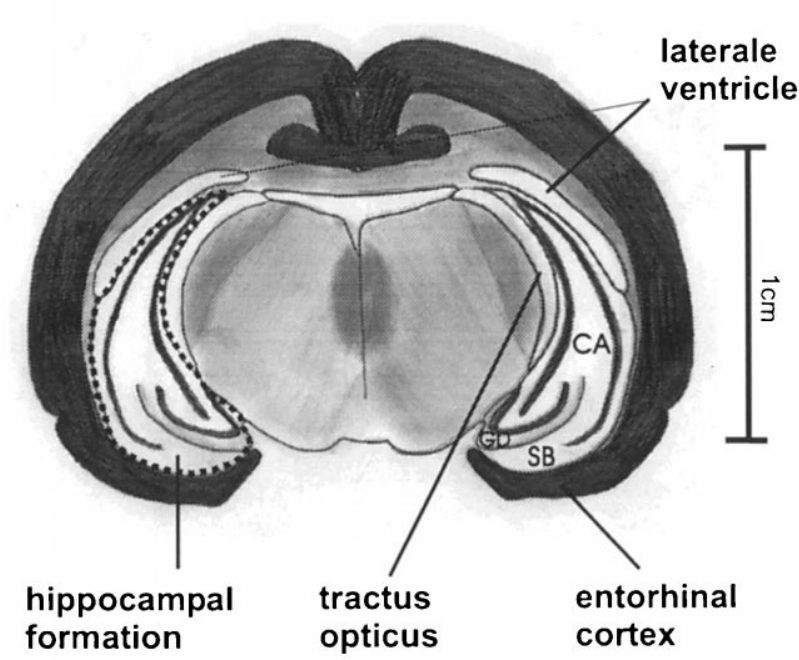

(b)

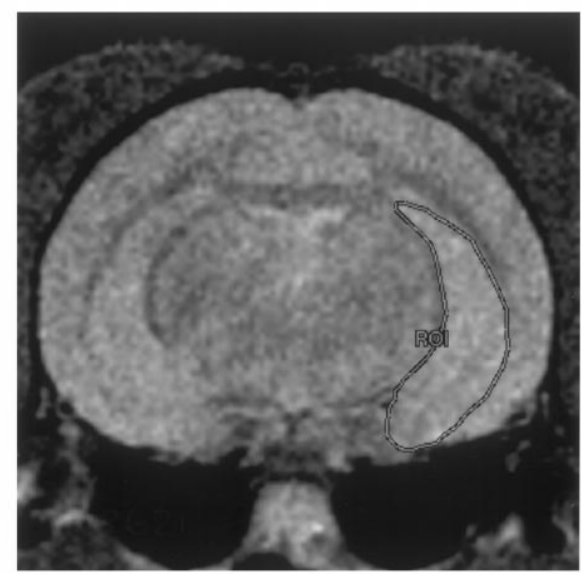

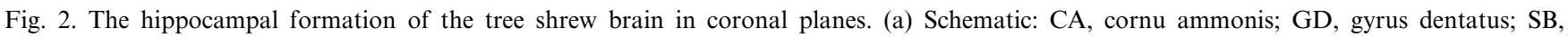
subiculum; (b) MR image (spin-echo, TR/TE = 3600/30 ms) marking the region-of-interest (ROI).

to ensure a proper positioning of the head. Finally, the midline sagittal gradient-echo image was employed to align coronal multi-slice spin-echo images (field of view $50 \mathrm{~mm}$, matrix $256 \times 256,25$ slices, section thickness 1 $\mathrm{mm}$ without a gap, two averages, $3600 \mathrm{~ms}$ repetition time, and echo times of 15,30 , as well as $45 \mathrm{~ms}$ ). For the anatomical analysis an echo-time of $30 \mathrm{~ms}$ resulted in best tissue contrast-to-noise ratio and was, therefore, used for the evaluation of the hippocampal volume of the tree shrew's brain.

\subsubsection{Volumetric measurement}

To assess the reproducibility and accuracy of the approach we evaluated the hippocampal area manually by manufacturer supplied software (ParaVision, Bruker, Karlsruhe, Germany). According to the tree shrew brain atlas by Tigges and Shantha (1969) six coronal sections (section thickness $1 \mathrm{~mm}$ ) ranging from AP 0.0 to AP 6.0 were measured. The coronal plane was preferred since both hippocampi can be evaluated simultaneously which enables comparative assessments of the left and right hemisphere. Images were analyzed on a computer monitor, using a mouse driven cursor. Left and right hippocampal formations were circumscribed by clear visual borders (Fig. $2 \mathrm{a}-\mathrm{b}$ ). The narrow strip of cerebrospinal fluid (lateral ventricle) marked the lateral and in parts the ventral borders of the hippocampus. The medial border was marked by the tractus opticus. Dorsally the fimbria hippocampi was included to maximum $10 \%$. Since a clear distinction between hippocampus and subiculum was not possible we included the subiculum to the measurements. Determination of the hippocampal volume $\left(\mathrm{mm}^{3}\right)$ was done by a region-of-interest (ROI) analysis.
To assess the intraindividual variability of the investigator, one animal was examined three times with an intersession interval of 2 weeks. One of these sessions was analyzed once on three different days by the same investigator. Additionally, to assess reproducibility of measurements and the interindividual variability of different investigators, the images of the three sessions were analyzed by three investigators each. To assess the interindividual variability of hippocampal volumes in tree shrews, we studied seven adult male individuals on one occasion each. All images of these sessions were analyzed by one investigator.

\subsubsection{Cortisol treatment}

To demonstrate the utility of MRI measurements for longitudinal studies male tree shrews $(n=3)$ were treated with cortisol via the drinking water (cortisol hemisuccinate, Steraloids, Wilton, NH, USA; $5 \mathrm{mg} /$ animal/day). Hippocampal volume was measured 4 weeks before the treatment, on the last day of the 4 weeks' cortisol treatment, 7 and 12 weeks after the treatment.

\section{Results}

Volumes of the hippocampal formation of male tree shrews (Table $1 ; n=7$ ) were determined as $85.2 \pm 6.7$ and $87.4 \pm 9.1 \mathrm{~mm}^{3}$ for the right and left hippocampus, respectively. The interindividual variability of the volumes was about $10 \%$ for both the right and left hippocampus.

The variability between repeated analysis of the same MRI session by one investigator (Table 2) was about $6 \%$. The right and left volumes of the tree shrew's 
Table 1

Left and right hippocampal volumes $\left(\mathrm{mm}^{3}\right)$ of seven adult male tree shrews

\begin{tabular}{lll}
\hline & \multicolumn{2}{l}{ Hippocampal formation } \\
\cline { 2 - 3 } & Left & Right \\
\hline Animal 1 & 90.4 & 93.1 \\
Animal 2 & 72.4 & 73.1 \\
Animal 3 & 87.5 & 98.1 \\
Animal 4 & 86.7 & 80.6 \\
Animal 5 & 92.5 & 87.7 \\
Animal 6 & 95.4 & 87.9 \\
Animal 7 & 81.7 & 80.8 \\
Mean \pm SD & $87.4 \pm 9.1$ & $85.2 \pm 6.7$ \\
\hline
\end{tabular}

Table 2

Hippocampal volume $\left(\mathrm{mm}^{3}\right)$ between repeated analysis of the same MRI session by the same investigator

\begin{tabular}{lll}
\hline & \multicolumn{2}{l}{ Hippocampal formation } \\
\cline { 2 - 3 } & Left & Right \\
\hline Analysis 1 & 86.8 & 89.8 \\
Analysis 2 & 83.8 & 79.9 \\
Analysis 3 & 82.3 & 75.7 \\
Mean \pm SD & $84.3 \pm 2.3$ & $81.8 \pm 7.2$ \\
\hline
\end{tabular}

hippocampus was determined as $81.8 \pm 7.2$ and $84.3 \pm$ $2.3 \mathrm{~mm}^{3}$, respectively.

Analysis of three MRI sessions by three investigators (Table 3 ) revealed variations of less than $5 \%$ over sessions in all investigators. Similarly, measurements over sessions in the same animal revealed variations below 5\%.

Chronic treatment with cortisol during 4 weeks days resulted in two of three animals in a clear reduction in hippocampal volume in the range of $10-15 \%$ (Fig. 3). Obviously, this reduction is reversible because 12 weeks after the treatment the volume tended to return to pretreatment values.

\section{Discussion}

The endotracheal intubation as well as the repeated

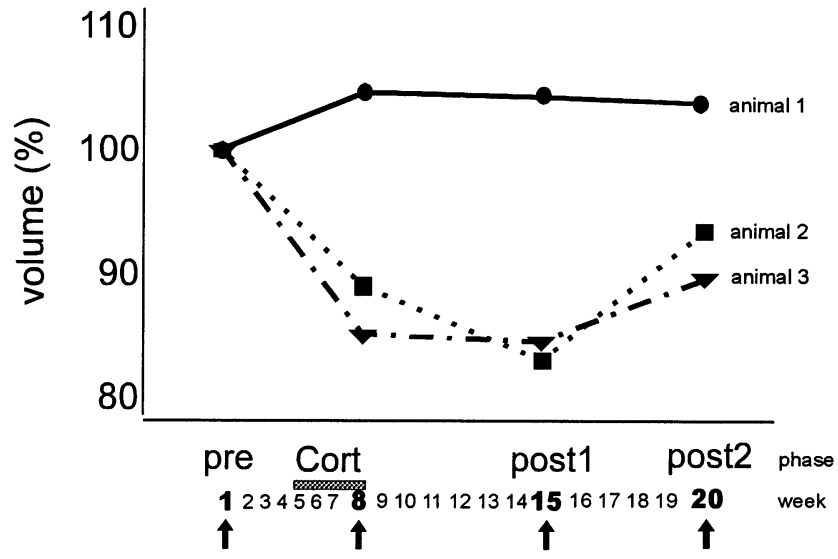

Fig. 3. Effect of chronic cortisol treatment on hippocampal volume in three tree shrews. Hippocampal volume was determined before (pre) and on the last day of a 4-week treatment with cortisol (Cort) via the drinking water $(5 \mathrm{mg} /$ day/animal). Volumes were determined 7 (post 1) and 12 weeks (post 2) after the cortisol treatment. Volumes are expressed as percent of the pretreatment values. Arrows indicate MRI sessions.

and prolonged anesthesia of about $2 \mathrm{~h}$ had no obvious effects on the behavior of the tree shrews. After termination of halothane supply the experimental animal recovered within 1-2 min.

The interindividual variability in male tree shrews was $10 \%$ for both the right and left hippocampal formation. The measurements showed highly reproducible results with variations below $6 \%$ between different investigators, within repeated MRI sessions of the same animal and also within repeated measurements of the same session by one investigator. This demonstrates the reliability of MRI measurements for in vivo investigations of the tree shrew's hippocampal volume.

In humans, neuroimaging techniques are frequently applied to patients suffering from psychoneuropathologies (Schacter, 1997). Although the cross-sectional approaches of these studies could bring up important information about particular states of neuropathologies, there is still a lack of information about the etiology and the temporal dynamics of these diseases being frequently accompanied by hippocampal atrophy (Jack et al., 1992; Fukuzako et al., 1996; O’Brian et al. 1996). Adequate experimental models might help to

Table 3

Left and right hippocampal volumes $\left(\mathrm{mm}^{3}\right)$ of one tree shrew

\begin{tabular}{|c|c|c|c|c|c|c|c|c|}
\hline & \multicolumn{2}{|c|}{ Investigator 1} & \multicolumn{2}{|c|}{ Investigator 2} & \multicolumn{2}{|c|}{ Investigator 3} & \multicolumn{2}{|c|}{ Mean \pm SD } \\
\hline & Left & Right & Left & Right & Left & Right & Left & Right \\
\hline Session 2 & 90.5 & 105.2 & 96.6 & 102.6 & 90.5 & 88.8 & $92.5 \pm 3.5$ & $98.9 \pm 8.8$ \\
\hline Session 3 & 86.1 & 92.4 & 84.5 & 87.2 & 90.8 & 90.9 & $87.1 \pm 3.3$ & $92.0 \pm 0.9$ \\
\hline Mean \pm SD & $89.0 \pm 2.5$ & $96.9 \pm 7.2$ & $91.8 \pm 6.4$ & $92.6 \pm 8.7$ & $90.8 \pm 0.4$ & $90.7 \pm 1.8$ & & \\
\hline
\end{tabular}

\footnotetext{
${ }^{a}$ The images of three sessions were analyzed by three independent investigators. The time interval between each sessions was 2 weeks.
} 
solve this question and tree shrews represent a potential animal model to mimic the temporal dynamics of stress-induced neuropathologies. Recently we reported that chronic psychosocial stress leads to structural alterations in the hippocampal formation of the tree shrew brain. After acute stress we observed a reduced proliferation rate of granular neurons in the dentate gyrus (Gould et al., 1997) and after chronic stress an involution of apical dendrites of pyramidal neurons in the CA3 area (Magariños et al., 1996). We also demonstrated, that stressful experiences resulted in long lasting memory impairments in the animals (Ohl and Fuchs, 1998).

The utility of MRI as a tool for neuroanatomical studies allowing repeated measurements of changes in soft tissue anatomy within the same animal throughout treatments over extensive time courses was demonstrated by investigating animals before and after application of cortisol via the drinking water. The daily dose of $5 \mathrm{mg}$ cortisol resulted in significantly elevated urinary cortisol levels being in a range similar to that of chronically stressed tree shrews (Isovich, pers. comm.). The observed cortisol-induced reduction of the hippocampal volume in two animals is in line with a recent study demonstrating in elderly humans a decrease in a similar range of $10-15 \%$ (Lupien et al., 1998). Interestingly, in the present experiment, the hippocampal shrinkage was still present 7 weeks after the last cortisol application and the volume tended to return to pre-treatment values 12 weeks after cortisol application. Though these data are from a small number of animals, the results reported here point to the future potentials and the forte of MRI as a non-invasive methodology for long-term neuroanatomical studies in experimental animals.

\section{References}

Eichenbaum H, Otto T, Cohen NJ. The hippocampus-What does it do? Behav Neural Biol 1992;57:2-36.

Flecknell PA. Laboratory Animals Anaesthesia. London: Academic Press, 1996.

Frahm J. Nuclear magnetic resonance studies of human brain in vivo: anatomy, function, and metabolism. Adv Exp Med Biol 1993;333:257-71.
Fuchs E, Schumacher M. Psychosocial stress affects pineal function in the tree shrew (Tupaia belangeri). Physiol Behav 1990;47:7137.

Fuchs E, Kramer M, Hermes B, Netter P, Hiemke C. Psychological stress in tree shrews: Clomipramine counteracts behavioral and endocrine changes. Pharmacol Biochem Behav 1996;54:219-28.

Fukuzako H, Fukuzako T, Takeuchi K, Ohbo Y, Ueyama K, Takigawa M, Fuijmoto T. Reduction in hippocampal formation volume is caused mainly by its shortening in chronic schizophrnia: assessment by MRI. Biol Psychol 1996;39:938-45.

Gould E, McEwen BS, Tanapat P, Galea LAM, Fuchs E. Neurogenesis in the dentate gyrus of the adult tree shrew is regulated by psychosocial stress and NMDA receptor activation. J Neurosci 1997; 17:2492-8.

Jack CR, Petersen RE, O'Brian PC, Tangalos EG. MR-based hippocampal volumetry in the diagnosis of Alzheimer's disease. Neurology 1992;42:183-8.

Lupien SJ, de Leon M, de Santi S, Convit A, Tarshish C, Nair NVP, McEwen BS, Hauger RL, Meaney MJ. Cortisol levels during human aging predict hippocampal atrophy and memory deficits. Nature Neurosci 1998;1:69-73.

Magariños AM, McEwen BS, Flügge G, Fuchs E. Chronic psychosocial stress causes apical dendritic atrophy of hippocampal CA3 pyramidal neurons in subordinate tree shrews. J Neurosci 1996;16:3534-40.

McEwen BS. Possible mechanisms for atrophy of the human hippocampus. Mol Psych 1997;2:255-62.

Morris TH, Jackson RK, Acker WA, Spencer CK, Drag MD. An illustrated guide to endotracheal intubation in small non-human primates. Lab Anim 1997;31:157-62.

O'Brian JT, Ames D, Schweitzer I, Colman P, Desmond P, Tress B. Clinical and magnetic resonance imaging correlates od hypothalamic-pituitary-axis function in depression and Alzheimer's disease. Br J Psychiatry 1996;168:679-87.

Ohl F, Fuchs E. Memory performance in tree shrews: effects of stressful experiences. Neurosci Biobehav Rev 1998;23:319-23.

Schacter DL. The cognitive neuroscience of memory: perspectives from neuroimaging research. Phil Trans R Soc London B 1997;52:1689-95.

Squire LR. Memory and the hippocampus: A synthesis from findings with rats, monkeys, and humans. Psychol Rev 1992;99:195-231.

Ter Laak HJ, Thijssen JM, Vendrik AJH. A method for prolonged electrophysical experiments with the tree shrew (Tupaia chinesis). Z Versuchstierkunde 1975;17:195-201.

Tigges J, Shantha TR. A Stereotaxic Brain Atlas of the Tree Shrew (Tupaia glis). Baltimore: Williams and Wilkins, 1969.

Ting Y-L, Bendel P. Thin-section MR imaging of rat brain at $4.7 \mathrm{~T}$. J Magn Reson Imaging 1992;2:393-9.

Tulving E, Markowitsch HJ. Memory beyond the hippocampus. Curr Opin Neurobiol 1997;7:209-16.

Vullo T, Deo-Narine V, Stallmeyer MJB, Gomez DG, Cahill PT. Quantitation of normal canine hippocampus formation volume: correlation of MRI with gross histology. Magn Reson Imaging 1996;14:657-62. 